\title{
A Greater increase in C5aR1-level at onset and a smaller decrease in IgG-level after recovery in severer COVID-19 patients: A new analysis of existing data with Ishida's t-test1 and t-test2
}

\section{Torao Ishida ( $\square$ ishida-t@suzuka-u.ac.jp )}

Suzuka University of Medical Science

Ken Takagi

Suzuka University of Medical Science

\section{Gui-feng Wang}

Suzuka University of Medical Science

Nobuyuki Tanahashi

Suzuka University of Medical Science

Jun Kawanokuchi

Suzuka University of Medical Science

Hisayo Takagi

Suzuka University of Medical Science

Yi Guo

Tianjin University of Traditional Chinese

Ning Ma

Suzuka University of Medical Science

\section{Research Article}

Keywords: covid-19, individual, C5aR1-levels, SARS-CoV-2, acute respiratory distress syndrome, receptor binding domain

Posted Date: August 11th, 2021

DOI: https://doi.org/10.21203/rs.3.rs-778916/v1

License: (c) (1) This work is licensed under a Creative Commons Attribution 4.0 International License.

Read Full License 
$1 \quad$ Original Research

2 Title:

3 A Greater increase in C5aR1-level at onset and a smaller decrease in

4 IgG-level after recovery in severer COVID-19 patients: A new analysis

$5 \quad$ of existing data with Ishida's t-test1 and t-test2

6 Authors: Torao Ishida* ${ }^{1,2}$, Ken Takagi ${ }^{1}$, Gui-feng Wang ${ }^{1}$, Nobuyuki Tanahashi ${ }^{1}$, Jun

$7 \quad$ Kawanokuchi $^{1}$, Hisayo Takagi $^{1}, \mathrm{Yi} \mathrm{Guo}^{2}$ and Ning $\mathrm{Ma}^{1}$

8 Author Affiliations:

$9 \quad{ }^{1}$ Project for Advanced Science, Suzuka University of Medical Science, Suzuka, Mie

10 510-0293, Japan

$11{ }^{2}$ College of Traditional Chinese Medicine, Tianjin University of Traditional Chinese

12 Medicine, Tianjin, 300193, China

13 Correspondence:

14 Torao Ishida, ishida-t@ suzuka-u.ac.jp, Project for Advanced Science, Suzuka

15 University of Medical Science, 1001-1 Kishioka, Suzuka, Mie 510-0293, Japan.

16 Telephon: +81-90-7695-8812, Fax: +81-59-381-2386 


\section{Abstract}

18 It is important to exactly identify the difference in the changes in a receptor C5aR1-

19 levels at onset and Immunoglobulin G (IgG)-levels after recovery between severe (acute

20 respiratory distress syndrome) and nonsevere (pneumonia) coronavirus disease 2019

21 patients to reduce the severity of the disease and prevent reinfection with the causative

22 virus. 2012 literature studies were selected from the PubMed Central@ databases with

23 keywords of (((covid-19) AND individual) NOT review) AND C5a / IgG. Three studies

24 showing individual expression levels of C5a/C5aR1 / antibody in the patients before

25 and after the passage of time without significance were selected by assessing the

26 literatures. We extracted dynamics where the slope values of the regression line

27 between the initial $(X)$ and changed level $(C)$ with passage of time were not zero

significantly ( $p<0.05$ ) using SAS-JMP-10. We examined the significance of $C$ in the

patients with Ishida's t-test 1 and that of the difference in $C$ between the severe and nonsevere patients with Ishida's t-test2, which used the values on the above regression

31 line as the expected values of $C$. These tests suggested a greater increase in C5aR1in nonsevere patients. 


\section{Introduction}

36 COVID-19 (Coronavirus disease 2019) is a new pandemic disease caused by infection with SARS-CoV-2 (severe acute respiratory syndrome coronavirus 2). C5a anaphylatoxin and its receptor C5aR1 play a key role in the initiation and maintenance of several inflammatory responses by recruiting and activating neutrophils and monocytes in the lungs ${ }^{1}$. It is important to know exactly the difference in changes in C5aR1 levels at onset and antibody, especially Immunoglobulin G (IgG), levels after recovery, between severe and nonsevere COVID-19 patients to reduce the severity of the disease and prevent reinfection with SARS-CoV-2. Carvelli et al. ${ }^{2}$ reported that there was no statistically significant change in the concentration of C5a desArg (complement C5a removal of the C-terminal arginine) in plasma, or \% C5aR1 (receptor for C5a)-expressing neutrophils and monocytes between $<72 \mathrm{~h}$ and days $5-10$ after the beginning of hospital care from pneumonia (with two-tailed Wilcoxon signed-rank tests $^{3}$ ) and ARDS (acute respiratory distress syndrome) patients with Kenward-Roger method $^{4,5}$. Chen et al. ${ }^{6}$ reported that there was no statistically significant change in the $\operatorname{IgM}, \operatorname{IgG}$, and $\operatorname{Ig}$ A titers specific to the spike protein between hospital discharge and follow-up visit between 21 days and 28 days after discharge among nonsevere and severe patients except anti-RBD (receptor binding domain) IgA and neutralization 
53 activities with paired $t$-test ${ }^{7}$ for normal distribution and Mann-Whitney $\mathrm{U}^{8}$ for non-

54 normal distribution. Yang et al. ${ }^{9}$ reported that there were no significant changes in the

55 levels of antibody against SARS-CoV-2 surface spike protein RBD in either recurrent-

56 positive or nonrecurrent-positive patients between one and two weeks postdischarge.

57 Additionally, there were no significant changes in the levels in recurrent-positive

58 patients between one week before recurrent-positive detection and the time of the

59 detection or between detection time and one week after detection with Mann-Whitney U

60 tests $^{8}$.

61 However, we found that some of these changes over time $(C)$ were dependent on their

62 initial levels $(X)$ and not suitable for analysis by conventional statistical tests. We

63 developed new statistical tests (Ishida's t-test1 and t-test2) that correctly examine the

64 above changes and then, we analyzed the above changes with Ishida's t-test1 and t-test2

65 to obtain different results from their papers as follows (research funding was not

66 provided by any organ. Approval Number of Clinical Ethics Committee in Suzuka

67 University of Medical Science is No. 322 (Judgment that it does not correspond to

68 clinical study). UMIN-CTR ID: UMIN000035831).

$69 \quad$ Results 

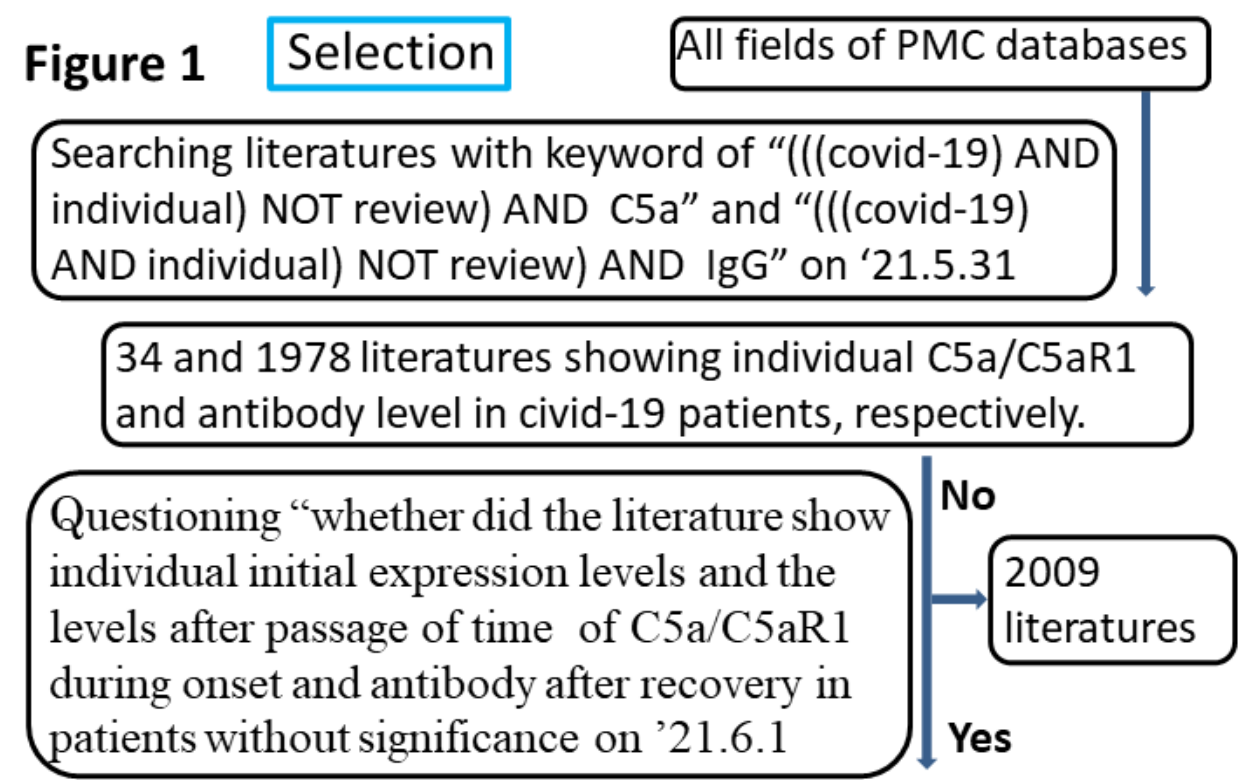

3 literatures ( Carvelli et $\mathrm{al}^{2}: 6$ dynamics, Chen et $\mathrm{al}^{6}: 13$ dynamics and 26 subdynamics, Yang et al $^{9}: 20$ dynamics). Analysis of $\beta$ (the slope value of $E$-line). See Fig. 2 and Extended Fig. 1. E-line was described in text.

\begin{tabular}{|l|l|}
\hline Extraction & $\begin{array}{l}p \geq 0.05 \text { dynamics } \\
\text { and their related } \\
p<0.05 \text { dynamics }\end{array}$ \\
\hline $\begin{array}{l}\beta \neq 0 \text { with } \\
p<0.05, ' 21,6.2\end{array}$ & $\begin{array}{l}\text { Remaining } \\
p<0.05 \text { dynamics }\end{array}$ \\
\hline
\end{tabular}

Chen et $a^{6}: 7$ dynamics and 14 subdynamics, Yang et $\mathrm{al}^{9}: 14$ dynamics. See Extended Table 1.

Carvelli et $\mathrm{al}^{2}: 6$ dynamics, Chen et $\mathrm{al}^{6}: 6$ dynamics and 12 sub dynamics, Yang et $\mathrm{al}^{9}: 6$ dynamics. See Table 1 and Table 2.

According to the procedure for selection of literatures described in Methods and in the first half of Figure 1, we selected one study on $\mathrm{C} 5 \mathrm{a} / \mathrm{C} 5 \mathrm{aR} 1^{2}$ and two studies on antibodies $^{6,9}$ in COVID-19 patients from 2012 literature studies selected from the PMC 
between $X$ and $C$ made by the method of least squares ( $E$-line) (Figure 2 and Extended Figure 1a and 1b) of 39 dynamics and 26 subdynamics (Table 1 and Extended Table 1) described in the three literatures. Extraction of dynamics dependent on $X$.

81 According to the procedure for extraction of dynamics described in Methods and in the latter half of Figure 1, we extracted eighteen dynamics and twelve subdynamics dependent on $X$ (Table 1). $C$-axis intercept $(\alpha)$, slope value $(\beta), X$-axis intercept $(\gamma(=-$ $\alpha / \beta))$ of E-line, standard error $(S E)$, standard deviation $(S D(=S E \sqrt{N}))$, mean of $C(M c)$, $\mathrm{p}$-value $(p)$, and effect size (Cohen's $\mathrm{d}^{10}(d(=|M c| / S D))$ for $\beta$ of extracted dynamics were shown on upper left of Table 2 . All of $\beta$ of $E$-line for 18 dynamics and 12 subdynamics were significantly $(p<0.05)$ negative. Thus, in these dynamics, $E$ was significantly inversely proportional to $X$ to the extent represented by $\mathrm{p}$-value for $\beta$ described on upper left of Table 2. value $(E)$ of $C, M e$, standard deviation $\left(S D_{1}\right)$, p-value $\left(p_{1}\right)$, effect size $\left(d_{1}\right)$ for $M e$ of the extracted dynamics with Ishida's t-test1 (upper right of Table 2). When $p_{1}<0.05, C$ also significantly inversely nearly proportional to $X$ to the extent represented by p-value for 
Me described on upper right of Table 2. In one dynamic (109) and five sub-dynamics (101s, 109n, 109s, 10yn and 10ws), the $E$ of all subjects decreased with passage of time from hospital discharge to days 21-28 after hospital discharge. However, in 13 dynamics $(081,091,092,093,094,101,107,108,10 y, 10 w, 211,212$ and 221) and 5 subdynamics (107n, 108n, 10ys, 10wn and 10ws), the $E$ of those subjects whose $X$ were less than $\gamma$ increased and those of other subjects decreased with passage of time significantly to the extent represented by $\mathrm{p}$-value for $M e$ described on upper right of

\section{Table 2.}

Generally, measuring of values before and after treatment included RTM (regression to the mean $)^{11}$. Therefore, the above $M e$ should subtract $M e$ of placebo control for the passage of time. However, no placebo control for passage of time was described in all of the three studies ${ }^{2,6,9}$ and others (33 studies on C5a/C5aR1 and 1976 studies on antibodies) described in Figure 1. Therefore, we could not get $M e$ free from $R T M^{11}$.

\section{Analysis of the extracted dynamics with Ishida's test2}

As we could not get $M e$ free from $R T M^{11}$, we analyzed the difference between $M e$ of particular group $k$ and $M e$ of particular group $l(d M e)$ under the assumption that at least one group is significantly different from $R T M^{11}$, if there is a significant difference between the two groups. 
113 We analyzed the difference between $d M e$, standard deviation $\left(S D_{2}\right)$, p-value $\left(p_{2}\right)$, effect

114 size $\left(d_{2}\right)$ for $d M e$ of the related two dynamics with Ishida's t-test2 (lower of Table 2).

115 The $\mathrm{d} M e$ of pneumonia (081, 091 and 093) and ARDS (082, 092 and 094) patients; and

116 nonsevere (107n - 109n and 10yn) and severe (107s - 109s and 10ys) patients was

117 significant to the extent represented by p-value for $d M e$ described in Table 2.

118 There was risk of bias due to limitation of databases, $R T M^{11}$ by double measurements,

119 limitation of numbers of subjects whose data were able to be estimated from the spots

120 or lines drawn in figures and its estimation errors, and limitation of validity of Ishida's

$121 \quad$ t-test1 and t-test2.

\section{Discussions}

123 The $E$-line crossed the $C$ axis at $\alpha$. When $\beta \neq 0$ with $\mathrm{p}<0.05$, this line also crossed the $X$

124 axis at $\gamma$. Thus, the equation for this line was described as $E=\beta(X-\gamma)$. A value obtained

125 from this equation in which $X i$ ( $X$ of particular subject $i$ ) was put was set as $E i$. As

126 deviation of $C i(C$ of particular subject $i)$ from $M c$ is $C i-M c$. Thus, that of $C i$ from $E i$

127 was set as $C i-E i$. The equation showed that $E$ was dependent on $X$. When $\beta \neq 0$ with p

$128<0.05$ and p-value of $M e$ was less than $0.05, C i$ exists on or near $E i$. Thus, $C$ was also

129 significantly nearly dependent on $X$. The $E$-line is made by the method of least squares. 
130 Thus, $\sum_{i=1}^{N}(C i-E i)^{2}$ was minimum and $\leq \sum_{i=1}^{N}(C i-M c)^{2}$. Thus, When $C$ was

131 nearly significantly dependent on $X$, the expected value of $C$ was not $M c$ but $E i$. For that

132 reason, Ishida's t-test1 was developed by replacing $\mathrm{Mc}$ and $[\mathrm{Ci}-\mathrm{Mc}]$ of paired t-test ${ }^{7}$

133 with $M e$ and $[C i-E i]$ and Ishida's t-test 2 was developed by replacing $d M c(M c$ of

134 particular group $k-M c$ of particular group $l)$ and $d[C i-M c]([C i-M c]$ of particular

135 group $k-[C i-M c]$ of particular group $l$ ) of unpaired t-tests (Student's t-test ${ }^{7}$, Welch's

136 t-test $^{12}$, and Tukey's multiple comparison test $\left.{ }^{13}\right)$ with $d M e$ and $d[C i-E i]([C i-E i]$ of

137 particular group $k-[C i-E i]$ of particular group $l$ ) under the condition that $C$ is

138 significantly nearly dependent on $X$. The following was demonstrated using 092

139 dynamics as an example.

140 Figure 2 showed the $E$ line for 092 (dynamics of \% C5aR1-expressing neutrophils in

141 ARDS patients between $<72 \mathrm{~h}$ and days 5-10 after the beginning of hospital care shown

142 in extended Fig. 2a 2nd from left by Carvelli et al. ${ }^{2}$ ).

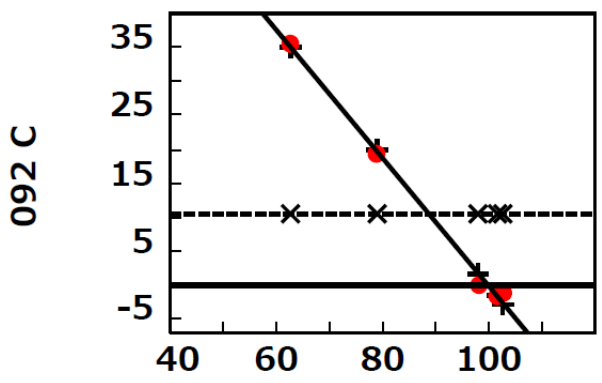

143 Figure 2.

$092 \times$ 
144 The $\beta$ for 092 was significantly negative $(p=0.0002)$ and $p_{1}$ for $M e$ was 0.00004 .

145 Figure 2 showed that $C$ existed on or near by the $E$-line and far from the $M c$ (Compare 146 the difference between "॰" and "+" and the difference between "॰" and " $x$ " in Figure

147 2.). Thus, $\sum_{i=1}^{N}(C i-E i)^{2}<\sum_{i=1}^{N}(C i-M c)^{2}$ in 092 . Thus, the expected value of $C i$

148 was $E i$ but not $M c$ and the expected value of $M c$ was $M e$ because $C$ of 092 was

149 significantly nearly dependent on $X$.

150 As previously mentioned, measuring values before and after the passage of time

151 included $\mathrm{RTM}^{11}$ but placebo control for the passage of time was not described in 2012

152 literatures on $\mathrm{C} 5 \mathrm{a} / \mathrm{C} 5 \mathrm{aR} 1$ and antibodies selected from the PMC databases. We should

153 plan clinical trials including placebo control for the passage of time, which will be

154 easily obtained by measuring marker values again immediately after measuring their

155 initial values.

156 Unlike the conclusions of Carvelli et al. ${ }^{2}$, analysis of their paper with Ishida's tests

157 suggested the following under the assumption that at least one group was significantly

158 different from $R T M^{11}$, as there was a significant difference between the two groups. 1)

159 C5a levels of pneumonia patients decreased significantly $(p=0.004)$ with a large effect

160 size $(d=0.77)$ but C5a levels of ARDS patients increased without significance $(p=$

$1610.068)$ and with a moderate effect size $(d=0.38)$ for at least 10 days after the beginning 
of hospital care. 2) There was significant $(p=0.002)$ difference in changes in C5a levels

163 between pneumonia patients and ARDS patients for the passage of time mentioned

164 above with large effect size $(d=1.01) .3)$ The \% C5aR1-expressing neutrophils of

165 pneumonia patients increased slightly but significantly $\left(p=3 \mathrm{e}^{-05}\right)$ with large effect

166 size $(d=9.78)$ but those of ARDS patients increased ten times more than those of

167 pneumonia patients significantly $\left(p=4 \mathrm{e}^{-05}\right)$ with a large effect size $(d=8.90)$ for the

168 passage of time mentioned above. 4) There was a significant ( $p=10 \mathrm{e}-08)$ difference in

169 the \% C5aR1-expressing neutrophils between pneumonia patients and ARDS patients

170 at the passage of time mentioned above, with a large effect size $(d=11.4) .5)$ The $\%$

171 C5aR1-expressing monocytes of pneumonia patients increased significantly $(p=$

$1720.0002)$ with a large effect size $(d=1.29)$ but those of ARDS patients increased two and

173 half times more than those of pneumonia patients significantly ( $p=7 \mathrm{e}-10)$ with large

174 effect size $(d=3.13)$ at the passage of time mentioned above. 6) There was a significant

$175\left(p=1 \mathrm{e}^{-05)}\right.$ difference in the \% C5aR1-expressing monocytes between pneumonia

176 patients and ARDS patients at the passage of time mentioned above, with a large effect

$177 \quad$ size $(d=1.87)$.

178 Unlike the conclusions of Chen et al. ${ }^{6}$, analysis of their paper with Ishida's t-test1

179 suggested that not only anti-RBD IgA (109) but also other antibodies (anti-RBD IgM 
180 (101), anti-S1 IgG (107), anti-NP (nucleoprotein) $\operatorname{IgG}(108)$, and anti-S1 IgA (10y))

181 reduced with the passage of time by each $M e$ with each p-value described on upper right

182 of Table 2 and these reduced antibodies might be responsible for the declining trend of

183 neutralizing activities (10w) by 1973 titers ( $p=3 \mathrm{e}-09$ with $d=3.10)$. Analysis with

184 Ishida's t-test2 suggested that anti-S1 IgG (107) and anti-NP IgG (108) of nonsevere

185 patients decreased more than those of severe patients $(\mathrm{p}=6 \mathrm{e}-07$ with $d=4.65$ and $\mathrm{p}=$

186 1e-09 with $d=8.23$ ), respectively, under the same assumption described above.

187 There were no significant differences in antibody levels between 211 and 212, between

188221 and 222, and between 229 and 22x with Ishida's t-test2. Our results could not be

189 differentiated from the conclusion of Yang et al. ${ }^{9}$, because we could not assuming that

190 at least one of the two groups compared was significantly different from $R T M^{11}$.

\section{Conclusion}

192 When clinical trials observing effects of passage of time will be planed, placebo control

193 for the passage of time and analysis of $\beta$ of the regression line between $X$ and $C$ should

194 be included. When $\beta \neq 0$ with $\mathrm{p}<0.05$, the clinical trials should be analyzed with

195 Ishida's t-test1 and t-test2.

\section{Methods}


197 Approval by the Clinical Research Ethics Review Committee:

198 Approval for the study was granted by the Clinical Research Ethics Review Committee

199 at Suzuka University of Medical Science (Approval No. No322, Approval date:

$200 \quad 2020 / 07 / 30)$.

201 Registration of study:

202 The study was registered in the UMIN Clinical Trials Registry (CTR ID:

203 UMIN000035831) as follows: Analysis of the effects of treatment with alternative

204 medicine and administration of medicine on the physiological value before treatment or

205 administration. The date of disclosure of the study information was 2019/02/12

206 Ethical Guidelines:

207 All experiments were performed in accordance with Ethical Guidelines for Medical and

208 Health Research Involving Human Subjects by Ministry of education and Ministry of

209 Health, Labor and Welfare in Japan and the Declaration of Helsinki.

210 Informed consent:

211 Our studies were only proposals concerning new t-tests and analysis of anonymized

212 data described in literatures concerning clinical studies with the t-tests without any 
213 contact with patients. In that case, "written informed consent" was not required by the

214 Ethical Guidelines or able to be obtained. Thus, the authors did not obtain written

215 informed consent from any participants.

\section{Data Sources:}

217 The data source of this meta-analysis is all field of the PMC databases (up to June $1^{\text {st }}$, 218 2021).

219 Selection of literatures:

220 We searched literatures with keyword of “(((covid-19) AND individual) NOT review)

221 AND C5a” and “(((covid-19) AND individual) NOT review) AND IgG”. Then, we

222 selected literatures obtained by keyword search with questioning "whether did the

223 literature show individual initial expression levels and the levels after passage of time of

$224 \mathrm{C} 5 \mathrm{a} / \mathrm{C} 5 \mathrm{aR} 1 \mathrm{during}$ onset and antibody after recovery in patients without significance.

225 Data Extraction and Synthesis:

$226 \alpha, \beta$, its $S E$, and its $p$-value of dynamics described in selected literatures were obtained

227 with SAS JMP 10 (Corporate Headquarters 100 SAS Campus Drive Cary, NC 27513,

228 USA). $\gamma, \mathrm{SD}$, and $d$ of the dynamics were obtained from $\gamma=-\alpha / \beta, S D=S E \sqrt{N})$, and $d$

$229=|M c| / \mathrm{SD}$, respectively, where $N$ indicated number of subjects. The dynamics where $\beta$ 
231 literatures.

232 Ishida's t-test1:

Ishida's t-test1 is a new two-tailed t-test fit for pair samples where $\beta \neq 0$ with $\mathrm{p}<0.05$.

following formulae were incorporated.

The $p_{1}$ were determined by inputting the number of degrees of freedom and $t_{1}$ into Excel 2019 T.DIST.2T function. A $p_{1}$ of $<0.05$ was considered statistically significant. $M p=\frac{1}{N} \sum_{i=1}^{N p}(E p i)$, where $N p=$ the numbers of subjects having $E p, E p i=E p$ of particular subject i, and $E p=$ positive expected value of $C$. $M n=\frac{1}{N} \sum_{i=1}^{N n}(E n i)$, where $N n=$ the numbers of subjects having En, Eni $=E n$ of particular subject i, and $E n=$ negative expected value of $C$.

$$
\begin{aligned}
& M e=\frac{1}{N} \sum_{i=1}^{N}(E i)=\frac{1}{N} \sum_{i=1}^{N p}(E p i)+\frac{1}{N} \sum_{i=1}^{N n}(E n i)=M p+M n \\
& S D_{1}=\left[\frac{1}{N-1} \sum_{i=1}^{N}(C i-E i)^{2}\right]^{\frac{1}{2}} \\
& S E_{1}=\left[\frac{1}{N-1} \sum_{i=1}^{N}(C i-E i)^{2}\right]^{\frac{1}{2}} / \sqrt{N} \\
& t_{1} \text {-value }=|M e| / S E_{1} \text { and } d_{1}=|M e| / S D_{1}
\end{aligned}
$$


247 Galton ${ }^{11}$. Thus, the marker values without the passage of time should be observed as

248 placebo control for $C$. $C$ should be cut off by the values of placebo control.

249 When there was no placebo for the changes with the passage of time, we examined the

250 significance of the difference between $C$ of the dynamics in patients in any two related

251 groups with Ishida's t-test2 described later to estimate significance of $C$ under the

252 assumption that at least one group is significantly different from $R T M^{11}$, if there is a

253 significant difference between the two groups.

254 Ishida's t-test2:

255 Ishida's t-test2 is a new two-tailed t-test fit for unpaired samples where $\beta \neq 0$ with $\mathrm{p}<$

256 0.05. $d M e, S D_{2}, S E_{2}, t_{2}$, and $d_{2}$ are calculated with using Excel where the following

257 formulae are incorporated.

number of groups and the number of subjects of a particular group $j$ respectively.

$$
S D_{2}=\left\{\sum_{j=1}^{m}\left[\sum_{i=1}^{N j}(C i-E i)^{2}\right]_{j} / \sum_{j=1}^{m}(N j-1)\right\}^{\frac{1}{2}}, \text { where } m \text { and } N j \text { indicate the }
$$

$$
\begin{aligned}
& S E_{2}=\left\{\sum_{j=1}^{m}\left[\sum_{i=1}^{N j}(C i-E i)^{2}\right]_{j} / \sum_{j=1}^{m}(N j-1)\right\}^{\frac{1}{2}} *\left[\frac{1}{N k}+\frac{1}{N l}\right]^{\frac{1}{2}} \\
& t_{2}=|d M e| / S E_{2} \text { and } d_{2}=|d M e| / S D_{2}
\end{aligned}
$$

263 When the number of groups was two, the $\mathrm{p}_{2}$-values were determined by inputting the 
number of degrees of freedom and $t_{2}$ into Excel 2019 T.DIST.2T function. When the

266 inputting the number of degrees of freedom, the number of groups, and $t_{2}$ into function

267 ">ptukey (t-value*sqrt(2), the number of groups, the number of degrees of

268 freedom,lower.tail=FALSE))" of open software R. A pd-value of $<0.05$ was considered

269 statistically significant.

270 Risk of bias in Methods:

271 There was risk of bias due to limitation of databases, $\mathrm{RTM}^{11}$ by double measurements,

272 limitation of numbers of subjects whose data were able to be estimated from the spots

273 or lines drawn in figures and its estimation errors, and limitation of validity of Ishida's

274 t-test1 and t-test2.

\section{$275 \quad$ References}

276 1. Wang, R., Xiao, H., Guo, R, Li, Y., Shen, B. The role of C5a in acute lung injury induced by highly pathogenic viral infections. Emerging microbes \& infections. 4(1), 1-7 (2015). https://doi.org/10.1038/emi.2015.28. 
2. Carvelli, J., et al. Association of COVID-19 inflammation with activation of the C5a-C5aR1 axis. Nature. 588(7836), 146-150 (2020). doi:10.1038/s41586-0202600-6.

3. Wilcoxon, F. Individual comparisons by Ranking Methods. Biometrics Bulletin. 1(6), 80-83 (1945). DOI: 10.2307/3001968.

4. Kenward, M. G. and Roger, J. H. Small sample inference for fixed effects from restricted maximum likelihood. Biometrics. 53(3), 983-997 (1997). https://doi.org/10.2307/2533558. https://www.jstor.org/stable/2533558.

5. Kenward, MG and Roger JH. An improved approximation to the precision of fixed effects from restricted maximum likelihood. Computational Statistics \& Data Analysis. 53(7), 2583-2595 (2009). https://doi.org/10.1016/j.csda.2008.12.013.

6. Chen, Y. et al. A comprehensive, longitudinal analysis of humoral responses specific to four recombinant antigens of SARS-CoV-2 in severe and non-severe COVID-19 patients. PLoS Pathogens. 16(9), e1008796 (2020). https://doi.org/10.1371/journal. ppat.1008796. 
7. Student (Gosset, W. S.). The probable error of a mean. Biometrika. 6(1), 1-25 (1908). https://www.jstor.org/stable/i315351. DOI:10.2307/2331554.

8. Mann, H. B. and Whitney, D. R. On a test of whether one of two random variables is stochastically larger than the other. Annals of Math. Stat. 18(1), 50-60 (1947).

9. Yang, C. et al. Viral RNA level, serum antibody responses, and transmission risk in Infections. 9, 2368-2378 (2020). https://doi.org/10.1080/22221751.2020.1837018

10. Cohen, J. Quantitative methods in psychology: A power primer. Psychological Bulletin. 112(1), 155-159 (1992). DOI: 10.1037/0033-2909.112.1.155.

11. Barnett, A. G., van der Pols, J. C. and Dobson, A. J. Regression to the mean: what it

12. Welch, B. L. On the comparison of several mean values: An alternative approach. Biometrika. 38(3/4):330-336 (1951). DOI: 10.2307/2332579. 
309 13. Tukey, J. W. Comparing individual means in the analysis of variance. Biometrics. 5(2): 99-114 (1959). DOI: $10.2307 / 3001913$.

\section{Acknowledgements}

312 T.I. thanks Mr. Jun-ichi Takagi, Chairman of SUMS for his financial support and Dr.

313 Zhang Boil, President of TUTCM for his encouragement.

\section{Author contributions statement}

315 T.I. designed two new statistical tests, run the study and wrote the manuscript. K.T.,

316 G.W., N.T., J.K., H.T., Y.G., N.M. contributed to discussions for the study. All authors

317 reviewed the manuscript.

\section{Additional information}

319 Competing interests

320 The authors declare no competing interests associated with this manuscript. Funding

321 was not supported.

Data availability

323 The authors declare that all data supporting the findings of this study are available

324 within the article and in a public (doi:10.1038/s41586-020-2600-6, 
326 https://doi.org/10.1080/22221751.2020.1837018) or from the corresponding author

327 upon reasonable request.

\section{Figure legend}

329 Figure1. Procedures for literatures selection and extraction of C5a/C5aR1 or antibody

330 dynamics in COVID-19 patients.

331 Figure 2. $E$-line for 092 . The solid diagonal line indicated $E$-line and that p-value of $\beta$

332 was $<0.05$. The dotted horizontal line indicated $M c$-line and that $\mathrm{p}$-value of $M c$ was $\geq$

$333 \quad 0.05 . \bullet,+$, and $\mathrm{x}$ indicated points at $(X i, C i),(X i, E i)$, and $(X i, M c)$, respectively. $E$-line,

$334 \quad 092, \beta, X i, C i, E i$, and $M c$ were described in text.

335 
Table 1. Contents of the included dynamics with their source.

081, Dynamics of concentration (ng/mL) of C5a desArg in plasma from pneumonia 082 (green: 081, $n=19$ ) and ARDS (red: 082, $n=25$ ) patients between T0 and T1 in extended Fig. $1 \mathrm{~b}$ by Carvelli et al. ${ }^{2}$

091 Dynamics of \% C5aR1-expressing neutrophils in pneumonia (green: 091, $\mathrm{n}=5$ )

092 and ARDS (red: 092, $\mathrm{n}=5$ ) patients between T0 and T1 in extended Fig. 2a by Carvelli et al. ${ }^{2}$

093 Dynamics of \% C5aR1-expressing monocytes in pneumonia (green: 093, $n=15$ )

094 and ARDS (red: 094, n=17) patients between T0 and T1 in extended Fig. 2a by

\begin{tabular}{l|l} 
& Carvelli et al. \\
\hline 101 & Dynamics of the \\
\hline
\end{tabular}

101 Dynamics of the IgM titer specific to RBD from non-severe (101n, $\mathrm{n}=15)$ and severe $(101 \mathrm{~s}, \mathrm{n}=5)$ patients between T3 and T4 in Fig. 6a anti-RBD IgM by Chen et al. ${ }^{6}$

107 Dynamics of the IgG titer specific to S1 from non-severe $(107 \mathrm{n}, \mathrm{n}=11)$ and severe $(107 \mathrm{~s}, \mathrm{n}=5)$ patients between T3 and T4 in Fig. 6a anti-S1 IgG by Chen et al. ${ }^{6}$

108 Dynamics of the IgG titer specific to NP from non-severe $(108 \mathrm{n}, \mathrm{n}=11)$ and severe 108 (108s, n=5) patients between T3 and T4 in Fig. 6a anti-NP IgG by Chen et al. ${ }^{6}$

109 Dynamics of the IgA titer specific to RBD from non-severe $(109 \mathrm{n}, \mathrm{n}=10)$ and severe \begin{tabular}{ll}
$(109 \mathrm{~s}, \mathrm{n}=5)$ patients between T3 and T4 in Fig. 6a anti-RBD IgA by Chen et al. \\
\hline $10 \mathrm{y}$
\end{tabular} \begin{tabular}{l|l}
$10 y$ & $\begin{array}{l}\text { Dynamics of the IgA titer specific to } S 1 \text { from non-severe }(10 y n, n=11) \text { and severc } \\
(10 y s, n=5) \text { patients between T3 and T4 in Fig. 6a anti-S1 IgA by Chen et al. }{ }^{6}\end{array}$ \\
\hline $10 \mathrm{~W}$ & Dyname
\end{tabular}

10w Dynamics of the NAb titer (IC50) from non-severe (10wn, $n=11$ ) and severe \begin{tabular}{l} 
(10ws, $n=5)$ patients between T3 and T4 described in Fig. 6a Nab by Chen et al. \\
\hline 211
\end{tabular} 211 Dynamics of IgM levels specific to RBD (titers) from non-recurrent-positive $212(211, n=35)$ and recurrent-positive $(212, n=6)$ patients between $\mathrm{T} 5$ and T6 described in Fig. 4a IgM level by Yang et al..$^{9}$

221 Dynamics of IgM levels specific to RBD (titers) from recurrent-positive patients 222 between T7 and T8 $(221, n=27)$ and between T8 and T9 $(222, n=23)$ described in Fig. 4c IgM level by Yang et al. ${ }^{9}$

229 Dynamics of NAb levels specific to RBD (titers) from recurrent-positive patients 22x between T7 and T8 $(229, \mathrm{n}=21)$ and between T8 and T9 $(22 \mathrm{x}, \mathrm{n}=18)$ described in Fig. 4c Nab level by Yang et al. ${ }^{9}$

$\mathrm{n}$; number of subjects, T0: $<72 \mathrm{~h}$ after the beginning of hospital care, T1: days 5-10 after the beginning of hospital care, T3: the time point of hospital discharge, T4: days 21-28 after hospital discharge, T5: week 1 hospital discharge, T6: weeks 2 hospital discharge, T7: one week before recurrent-positive detection, T8: the time point of recurrent-positive detection, T9: one week after recurrent-positive detection, ARDS: acute respiratory distress syndrome, C5a des-Arg: Complement C5a removal of the Cterminal arginine, C5aR1: receptor for the C5a anaphylatoxin; S1, NP, and RBD: S1 protein, nucleoprotein, receptor binding domain of the spike proteins of SARS-CoV-2; Ig: Immunoglobulin, and NAb: neutralizing antibody. 
Table 2. Analysis of the included dynamics in three studies ${ }^{2,6,9}$ with regression line and Ishida's t-test1 and t-test2.

\begin{tabular}{|c|c|c|c|c|c|c|c|c|c|c|c|}
\hline Grp & $\beta$ & $\gamma$ & $S D$ & $p$ & $d$ & $M p$ & $M n$ & $M e$ & $S D_{1}$ & $p_{1}$ & $d_{1}$ \\
\hline 081 & -0.61 & 47.0 & 0.51 & $<.0001$ & 1.19 & 2.60 & -13.91 & -11.30 & 14.77 & 0.004 & 0.77 \\
\hline 082 & -0.87 & 81.1 & 1.11 & 0.0007 & 0.79 & 12.64 & -3.89 & 8.75 & 22.88 & 0.068 & 0.38 \\
\hline 091 & -0.36 & 101.5 & 0.05 & 0.0005 & 7.12 & 1.02 & -0.02 & 1 & 0.10 & $3 e-05$ & 9.78 \\
\hline 092 & -0.94 & 99.9 & 0.09 & 0.0002 & 10.9 & 11.29 & -0.87 & 10.42 & 1.17 & $4 \mathrm{e}-05$ & 8.90 \\
\hline 093 & -0.65 & 94.6 & 0.40 & $<.0001$ & 1.61 & 5.73 & -0.41 & 5.32 & 4.11 & $2 \mathrm{e}-04$ & 1.29 \\
\hline 094 & -0.86 & 94.1 & 0.24 & $<.0001$ & 3.62 & 13.73 & -0.67 & 13.06 & 4.17 & $7 \mathrm{e}-10$ & 3.13 \\
\hline 101 & -0.81 & $3 \times 10^{4}$ & 0.71 & 0.0007 & 1.15 & 8381 & $-8 \times 10^{4}$ & $-7 \times 10^{4}$ & $1 \times 10^{5}$ & 0.017 & 0.70 \\
\hline $101 \mathrm{n}$ & -0.87 & $4 \times 10^{4}$ & 0.98 & 0.0162 & 0.89 & $1 \times 10^{4}$ & $-7 \times 10^{4}$ & $-5 \times 10^{4}$ & $1 \times 10^{5}$ & 0.174 & 0.44 \\
\hline $101 \mathrm{~s}$ & -0.72 & -9583 & 0.11 & 0.0053 & 6.87 & 0 & $-1 \times 10^{5}$ & $-1 \times 10^{5}$ & $2 \times 10^{4}$ & $6 e-04$ & 7.68 \\
\hline 107 & -1.01 & $4 \times 10^{4}$ & 0.09 & $<.0001$ & 11.3 & $2 \times 10^{4}$ & $-4 \times 10^{5}$ & $-3 \times 10^{5}$ & $8 \times 10^{4}$ & $3 e-11$ & 4.25 \\
\hline $107 \mathrm{n}$ & -1.00 & $1 \times 10^{4}$ & 0.02 & $<.0001$ & 60.9 & 5310 & $-5 \times 10^{5}$ & $-4 \times 10^{5}$ & $2 \times 10^{4}$ & $1 \mathrm{e}-15$ & 25.77 \\
\hline $107 \mathrm{~s}$ & -1.18 & $1 \times 10^{5}$ & 0.68 & 0.0306 & 1.73 & $6 \times 10^{4}$ & $-2 \times 10^{5}$ & $-1 \times 10^{5}$ & $1 \times 10^{5}$ & 0.128 & 0.86 \\
\hline 108 & -1.00 & $2 \times 10^{5}$ & 0.05 & $<.00$ & 19.5 & $7 \times 10^{4}$ & $-2 \times 10^{6}$ & $-2 \times 10^{6}$ & $4 \times 10^{5}$ & $1 \mathrm{e}-12$ & 6.13 \\
\hline $108 \mathrm{n}$ & -1.00 & $2 \times 10^{5}$ & 0.05 & $<.00$ & \begin{tabular}{ll|}
18.9 \\
\end{tabular} & $1 \times 10^{5}$ & $-3 \times 10^{6}$ & $-3 \times 10^{6}$ & $4 \times 10^{5}$ & $2 \mathrm{e}-09$ & 7.38 \\
\hline $108 \mathrm{~s}$ & -1.00 & $1 \times 10^{5}$ & 0.60 & 0.0 & 1.67 & $2 \times 10^{4}$ & $-2 \times 10^{5}$ & $-2 \times 10^{5}$ & $1 \times 10^{5}$ & 059 & 1.17 \\
\hline 109 & -0.42 & -306 & 0.17 & $<.0$ & 2.47 & 0 & -3260 & -3260 & 2125 & e-05 & 1.53 \\
\hline $109 n$ & -0.23 & -1346 & 0.27 & 0.0 & 0.83 & 0 & -1127 & -1127 & 11 & 0.014 & 0.97 \\
\hline $109 \mathrm{~s}$ & -0.41 & -3275 & 0.18 & 0.01 & 2.32 & 0 & -7526 & -7526 & 2960 & 0.005 & 2.54 \\
\hline $10 \mathrm{y}$ & -0.82 & 90.2 & 0.28 & $<.00$ & 2.96 & 8.24 & $-2 \times 10^{4}$ & $-2 \times 10^{4}$ & 9263 & $2 \mathrm{e}-06$ & 1.90 \\
\hline $10 \mathrm{yn}$ & -0.70 & -52.0 & 0.28 & $<.000$ & 2.55 & 0 & $-1 \times 10^{4}$ & $-1 \times 10^{4}$ & 8674 & $8 \mathrm{e}-04$ & 1.43 \\
\hline $10 \mathrm{ys}$ & -1.01 & 1303 & 0.03 & $<.00$ & 39.7 & 228 & $-3 \times 10^{4}$ & $-3 \times 10^{4}$ & 781 & $1 \mathrm{e}-07$ & 37.35 \\
\hline $10 \mathrm{w}$ & -0.94 & 546 & 0.13 & $<.00$ & 7.28 & 120 & -2093 & -1973 & 37 & $3 e-09$ & 3.10 \\
\hline $10 \mathrm{wn}$ & -0.94 & 679 & 0.13 & $<.0001$ & 7.27 & 254 & -2380 & -2126 & 748 & $3 e-06$ & 2.84 \\
\hline $10 \mathrm{ws}$ & -0.93 & 255 & 0.04 & $<.0001$ & 25.2 & 0 & -1636 & -1636 & 42 & $1 \mathrm{e}-07$ & 38.92 \\
\hline 211 & -0.26 & 0.87 & 0.16 & $<.00$ & 1.66 & 0.04 & -0.56 & -0.53 & 0.57 & $4 \mathrm{e}-06$ & 0.92 \\
\hline 212 & -0.34 & 0.72 & 0.17 & 0.00 & 2.06 & 0.06 & -0.72 & -0.67 & 0. & 0.006 & 1.85 \\
\hline 221 & -0.23 & 0.33 & 0.11 & $<.0001$ & 2.14 & 0.01 & -0.12 & -0.11 & 0.11 & $1 \mathrm{e}-05$ & 1.04 \\
\hline 222 & -0.45 & 0.94 & 0.77 & 0.0 & 0.58 & 0.22 & -0.13 & 0.10 & 0.82 & 0.579 & 0.12 \\
\hline 229 & -0.27 & 46.1 & 0.30 & 0.0047 & 0.70 & 5.33 & -18.13 & -12.80 & 57.92 & 0.323 & 0.22 \\
\hline $22 x$ & -0.80 & 69.57 & 0.29 & $<.0001$ & 2.78 & 38.65 & -53.97 & -15.32 & 70.12 & 0.367 & 0.22 \\
\hline Gr k & Gr I & $d M e$ & $S D_{2}$ & $p_{2}$ & $d_{2}$ & Gr k & Gr I & $d M e$ & $S D_{2}$ & $p_{2}$ & $d_{2}$ \\
\hline 081 & 082 & -20.1 & 19.8 & 0.002 & 1.01 & $109 n$ & $109 \mathrm{~s}$ & 6399 & 1908 & $4 \mathrm{e}-05$ & 3.35 \\
\hline 091 & 092 & -9.42 & 0.83 & $1 \mathrm{e}-07$ & 11.4 & $10 \mathrm{yn}$ & $10 \mathrm{ys}$ & $2 \times 10^{4}$ & 7343 & $8 \mathrm{e}-04$ & 2.72 \\
\hline 093 & 094 & -7.74 & 4.14 & $1 \mathrm{e}-05$ & 1.87 & 10wn & $10 \mathrm{ws}$ & -489 & 633 & 0.173 & 0.77 \\
\hline $101 n$ & $101 \mathrm{~s}$ & $7 \times 10^{4}$ & $1 \times 10^{5}$ & 0.276 & 0.66 & 211 & 212 & 0.14 & 0.55 & 0.559 & 0.26 \\
\hline $107 \mathrm{n}$ & $107 \mathrm{~s}$ & $-3 \times 10^{5}$ & $7 \times 10^{4}$ & $6 \mathrm{e}-07$ & 4.65 & 221 & 222 & -0.21 & 0.56 & 0.198 & 0.37 \\
\hline $108 n$ & $108 \mathrm{~s}$ & $-3 \times 10^{6}$ & $4 \times 10^{5}$ & $1 \mathrm{e}-09$ & 8.23 & 229 & $22 x$ & 2.52 & 63.8 & 0.903 & 0.04 \\
\hline
\end{tabular}

Abbr.: $C$-axis intercept $(\alpha)$, slope value $(\beta), X$-axis intercept $(\gamma(=-\alpha / \beta))$ of the regression line between the initial $(X)$ and changed $(C)$ value with treatment of a maker made by the method of least squares ( $E$-line); positive $(M p)$ and negative $(\mathrm{Mn})$ comportment of mean of expected value of $C(\mathrm{Me})$; difference between $\mathrm{Me}$ of Group (Grp) k and Group (Grp) 1 ( $d M e$ ); standard deviation and p-value of conventional $(S D, p)$, Ishida's t-test1 $\left(S D_{1}, p_{1}\right)$, t-test2 $\left(S D_{2}, p_{2}\right) ; \mid M c$ (mean of $C)|/ S D(d)| M e,\left|/ S D_{1}\left(d_{1}\right),\right| d M e \mid / S D_{2}\left(d_{2}\right)$; Contents and source of 081 - 22x were described in Table 1. Unit of $M p, M n, M e$, and $d M e: \mathrm{ng} / \mathrm{mL}(081,082), \%$ (091, 092, 093, 094), titers for IC50 (10w, 10wn, 10ws, 229, 22x), titers (Others). 


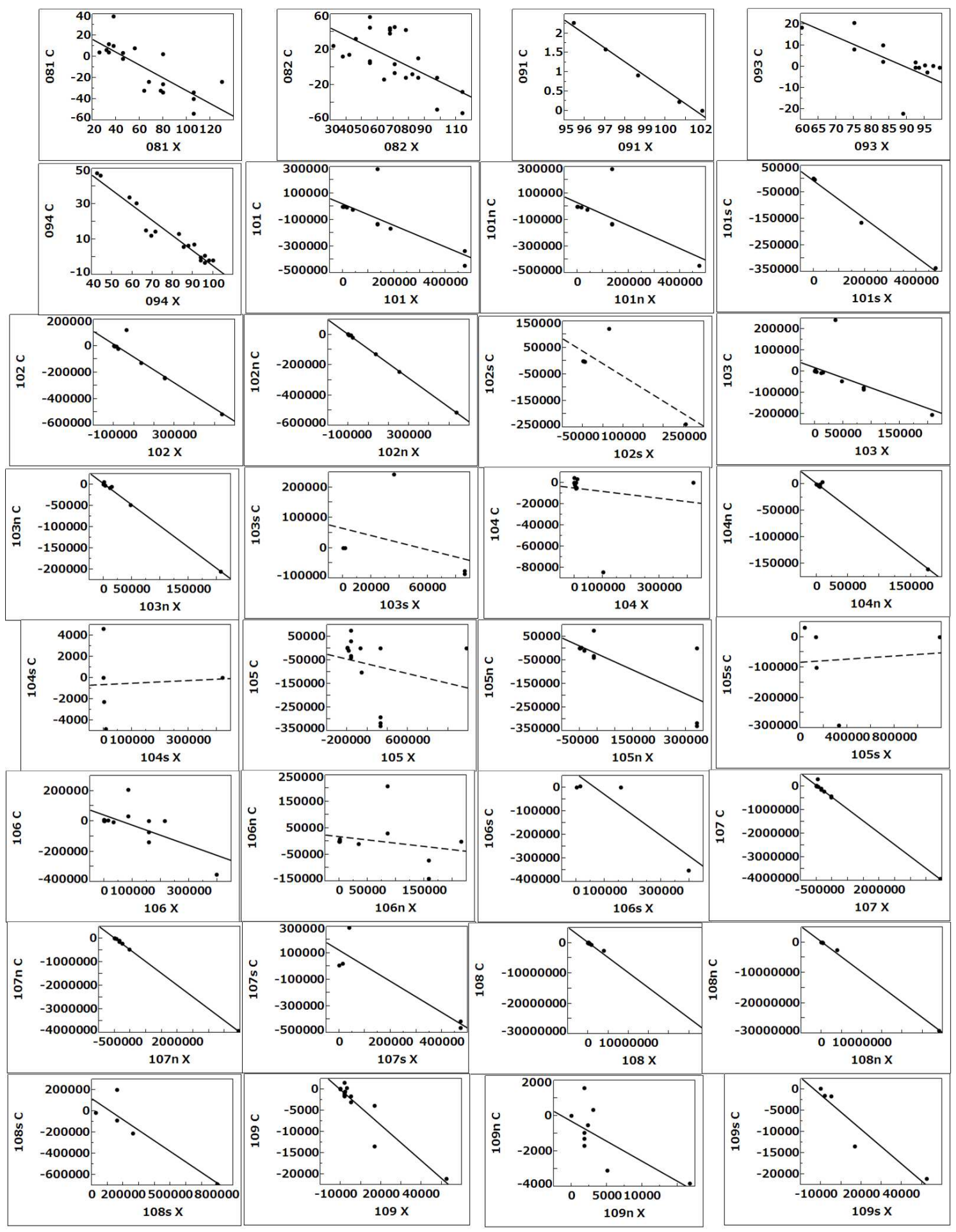

Extended Figure 1a. E-line for $091-109$ s. Contents of the dynamics with their source 
343 were described in Table 1 or Extended Table 1. Solid and dotted lines indicated that p-

344 value of slope of $E$-line was $<0.05$ and $\geq 0.05$, respectively.

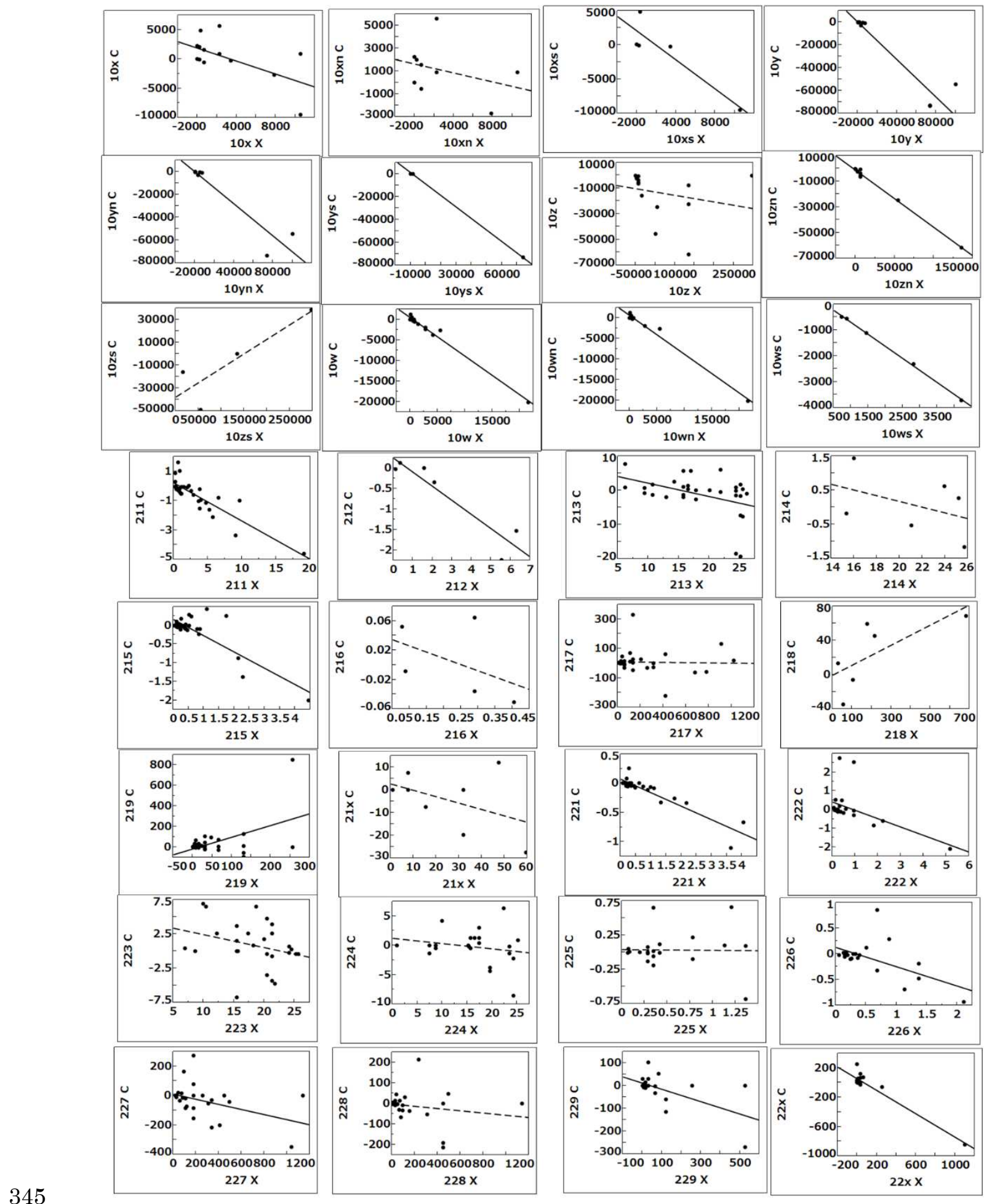


346 Extended Figure 1b. $E$-line for 10x $-22 x$. Contents of the dynamics with their source

347 were described in Table 1 or Extended Table 1. Solid and dotted lines indicated that p-

348 value of slope of $E$-line was $<0.05$ and $\geq 0.05$, respectively.

349 Extended Table 1. Contents of the excluded dynamics with their source.

102 Dynamics of the IgM titer specific to ECD from non-severe $(102 n, n=11)$ and severe $(102 s, n=5)$ patients between T3 and T4 in Fig. 6a anti-ECD IgM by Chen et al. ${ }^{6}$

103 Dynamics of the IgM titer specific to $S 1$ from non-severe $(103 n, n=11)$ and severe $(103 \mathrm{~s}, \mathrm{n}=5)$ patients between T3 and T4 in Fig. $6 \mathrm{a}$ anti-S1 IgM by Chen et al. ${ }^{6}$

104 Dynamics of the IgM titer specific to NP from non-severe $(104 n, n=11)$ and severe $(104 \mathrm{~s}, \mathrm{n}=5)$ patients between T3 and T4 in Fig. 6a anti-NP IgM by Chen et al. ${ }^{6}$

105 Dynamics of the IgG titer specific to RBD from non-severe $(105 n, n=11)$ and severe $(105 \mathrm{~s}, \mathrm{n}=5)$ patients between T3 and T4 in Fig. $6 \mathrm{a}$ anti-RBD IgG by Chen et al. ${ }^{6}$

106 Dynamics of the IgG titer specific to ECD from non-severe (106n, $n=11)$ and severe $(106 \mathrm{~s}, \mathrm{n}=5)$ patients between T3 and T4 in Fig. $6 \mathrm{a}$ anti-ECD IgG by Chen et al. ${ }^{6}$

10x Dynamics of the IgA titer specific to ECD from non-severe $(10 \mathrm{xn}, \mathrm{n}=9)$ and severe (10xs, $n=5)$ patients between T3 and T4 in Fig. 6a anti-ECD IgA by Chen et al. ${ }^{6}$

$10 \mathrm{z}$ Dynamics of the IgA titer specific to NP from non-severe (10zn, $\mathrm{n}=11)$ and severe (10zs, $n=5)$ patients between T3 and T4 in Fig. 6a anti-NP IgA by Chen et al. ${ }^{6}$

213 Dynamics of IgG levels specific to RBD (titers) from non-recurrent-positive

$214(213, \mathrm{n}=32)$ and recurrent-positive $(214, \mathrm{n}=6)$ patients between T5 and T6 described in Fig. 4 a IgG level by Yang et al. ${ }^{9}$

215 Dynamics of IgA levels specific to RBD (titers) from non-recurrent-positive

$216(215, \mathrm{n}=32)$ and recurrent-positive $(216, \mathrm{n}=5)$ patients between T5 and T6 described in Fig. 4a IgA level by Yang et al. ${ }^{9}$

217 Dynamics of all Ig levels specific to RBD (titers) from non-recurrent-positive

$218(217, \mathrm{n}=33)$ and recurrent-positive $(218, \mathrm{n}=6)$ patients between T5 and T6 described in Fig. 4a all Ig level by Yang et al. ${ }^{9}$

219 Dynamics of Nab levels specific to RBD (titers) from non-recurrent-positive

21x $(217, n=33)$ and recurrent-positive $(218, n=6)$ patients between T5 and T6 described in Fig. 4a Nab level by Yang et al. ${ }^{9}$

223 Dynamics of IgG levels specific to RBD (titers) from recurrent-positive patients

224 between T7 and T8 $(223, n=27)$ and between T8 and T9 $(224, n=23)$ described in Fig. 4c IgG level by Yang et al. ${ }^{9}$

225 Dynamics of IgA levels specific to RBD (titers) from recurrent-positive patients

226 between T7 and T8 $(225, \mathrm{n}=21)$ and between T8 and T9 $(226, \mathrm{n}=20)$ described in Fig. 4c IgA level by Yang et al. ${ }^{9}$

227 Dynamics of all Ig levels specific to RBD (titers) from recurrent-positive patients

228 between T7 and T8 $(227, \mathrm{n}=27)$ and between T8 and T9 $(228, \mathrm{n}=25)$ described in Fig. $4 \mathrm{c}$ all Ig level by Yang et al. ${ }^{9}$

Each abbreviation was described in Table 1. 